$(p<0.0001)$. No AEs occurred with CAN, only in one case $(1 / 4,25 \%)$ treatment was stopped for loss of efficacy.

Conclusions: To our knowledge this is the largest retrospective observational study evaluating the efficacy and safety of ANK and CAN in AOSD. A prompt response was demonstrated already at 3 months of follow-up both in ANK and CAN groups. ANK proved to be effective in both patterns of disease, appearing also safe on the infectious risk. Nonetheless, skin reaction may represent a not negligible AEs during ANK treatment.

Disclosure of Interest: None declared

DOI: 10.1136/annrheumdis-2017-eular.6166

\section{THU0542 ANTI-INTERLEUKIN 1 THERAPY IN FMF AMYLOIDOSIS: A SINGLE CENTER EXPERIENCE (CASE SERIES)}

S. Ugurlu, B. Ergezen, A. Hacioglu, H. Ozdogan. Division of Rheumatology, Department of Internal Medicine, Cerrahpasa Medical Faculty, University of Istanbul, Istanbul, Turkey

Background: Recently there is increasing number of reports investigating the efficacy of anti-interleukin-1 (anti-IL1) therapy in AA-amyloidosis.

Objectives: Here we report our experience in $\mathrm{IL}-1$ blockade in $\mathrm{AA}$ amyloidosis secondary to FMF.

Methods: Twenty nine FMF patients with secondary AA-amyloidosis with insufficient response to colchicine were treated with anti-IL-1 agents (canakinumab and anakinra). Creatinine (Cre), 24-hour urine protein (UP), erythrocyte sedimentation rate $(E S R)$ and $C$-reactive protein $(C R P)$ were measured before and throughout the treatment to evaluate the response and side effects.

Results: Twenty nine (16 female, 13 male) patients with FMF-related amyloidosis were administered anti-IL1 agents (12 on canakinumab, 17 on anakinra) in addition to colchicine in 27 patients, with a mean dose of $1.4 \pm 0.6 \mathrm{mg} /$ day. The mean age was $40.13 \pm 11.76$, while the mean duration of FMF was $28.79 \pm 10.51$ years. The mean follow-up was $13.92 \pm 11.31$ months for anakinra and $11.82 \pm 9.92$ months for canakinumab.

Initial Cre levels were less than $1.5 \mathrm{mg} / \mathrm{dl}$ in 13 patients (range 0.37-.5). In this subgroup proteinuria decreased significantly from $3739.87 \pm 4860.41$ to $1321.45 \pm 2015.62 \mathrm{mg} / \mathrm{day}$ while Cre was stable $(0.91 \pm 0.30 \mathrm{mg} / \mathrm{dl}$ to $1.04 \pm 0.39$ $\mathrm{mg} / \mathrm{dl}$ ) and acute phase response was normalized (CRP from $6.16 \pm 7.86 \mathrm{mg} / \mathrm{l}$ to $5.20 \pm 9.64 \mathrm{mg} / \mathrm{l}$, ESR from $27.3 \pm 18.63$ to $14 \pm 8.04)$.In the second group there were 11 patients with initial Cre levels higher than $1.5 \mathrm{mg} / \mathrm{dl}$ (range 1.73-3.76). Proteinuria decreased from $6321.66 \pm 5936.43$ to $4827.55 \pm 6264.43 \mathrm{mg} /$ day, Cre increased from $2.53 \pm 0.76$ to $3.07 \pm 1.70 \mathrm{mg} / \mathrm{dl}$, while there was a decrease in the APR in this subgorup (CRP from $40 \pm 64.65 \mathrm{mg} / \mathrm{l}$ to $26.71 \pm 26.76 \mathrm{mg} / \mathrm{l}$, ESR from $57.37 \pm 37.65$ to $39.11 \pm 27.72$ ).

There are two patients on hemodialysis whereas two underwent renal transplantation. Global patient assessment score of the whole group was decreased from $7.44 \pm 2.54$ to $3.89 \pm 3.53$ with anti-IL-1

Anakinra was stopped in 11 and canakinumab in 3 patients, due to irresponsiveness in 8 and 2 patients respectively. Among patients in whom anakinra was terminated 9 were later treated with canakinumab. Twenty four patients are still recieving anti-IL-1treatment (16 on anakinra, 8 on canakinumab).

Conclusions: Anti IL-1 treatments seems to be effective and safe in the treatment of AA amyloidosis secondary to FMF. The beneficial effect of this approach is more pronounced in patients with creatinine levels of less that $1.5 \mathrm{mg} / \mathrm{dl}$.

Disclosure of Interest: None declared

DOI: 10.1136/annrheumdis-2017-eular.5397

\section{THU0543 A RETROSPECTIVE OVERVIEW OF THE TREATMENT MODALITY, OUTCOME AND RELAPSE RISK OF IGG4 RELATED DISEASE IN HONG KONG: A DATASET OF 108 PATIENTS FROM FOUR REGIONAL HOSPITALS}

C.P. Tang ${ }^{1}$, K.L. Lee ${ }^{1}$, K.W. Lee ${ }^{2}$, W.L. $\mathrm{Ng}^{3}$, M.C. Wan ${ }^{4}$, K.Y. Yuen ${ }^{5}$. ${ }^{1}$ Medicine, PYNEH; ${ }^{2}$ Medicine, HKSH; ${ }^{3}$ Medicine, UCH; ${ }^{4}$ Medicine, $\mathrm{RH} ;{ }^{5}$ Medicine, QMH, HK, Hong Kong

Background: Currently most consensus suggests treating lgG4 related disease (lgG4RD) with prednisolone $0.6 \mathrm{mg} / \mathrm{kg} /$ day tapering over 6 months while some experts prefer continuation of steroid up to 3 years $(1-3)$.

Objectives: In this retrospective study, the treatment modality, response and relapse risk of IgG4RD patients over the past ten years from four regional hospitals in Hong Kong were analyzed.

Methods: Four regional hospitals participated with study period from 1/1/2006 to $30 / 6 / 2016$. Patients were diagnosed IgG4RD according to the Japanese Comprehensive Diagnostic Criteria for IgG4RD. Treatment response at 6 months was recorded as complete, partial or non-remission based on the patient's and physician's perspective. Relapse was defined as disease progression either clinically or radiologically

Statistical analysis: The association between treatment response and steroid dosage was analyzed by multivariate logistic regression. Risk factors for relapse were analyzed by multivariate cox regression and the hazard ratio was reported. Results: 37 cases had surgical excision and disease recurred in 5 cases (relapse rate $=13.5 \%$ ). 87 patients $(81 \%)$ received steroid treatment. The mean starting prednisolone was $33.5 \mathrm{mg}$ daily, with a mean duration of 95.2 weeks. At 6 months,
5 patients $(6 \%)$ had no response, 34 patients $(41 \%)$ had partial remission and 44 patients $(53 \%)$ had complete remission.

29 patients also received other immunosuppressants, including azathioprine $(n=25)$, mycophenolate mofetil (MMF) $(n=6)$, cyclosporine $A(n=4)$, mercaptopurine $(n=2)$ and rituximab $(n=1)$. The overall response rate for azathioprine and MMF was $64 \%$ and $67 \%$ respectively.

In the final multiple logistic regression, an initial prednisolone $30 \mathrm{mg}$ daily or above was associated with a higher complete remission at 6 months $(O R=3.4, p=0.079)$ and the effect was more seen in patients with salivary and orbital involvement $(\mathrm{OR}=6.8, \mathrm{p}=0.10)$

18 patients relapsed after steroid was stopped and 6 patients relapsed while on prednisolone $2.5-7.5 \mathrm{mg}$ daily. The one year, two year and three year relapse rate were $13.3 \%, 24.1 \%$ and $26.5 \%$ respectively.

In the final multivariate cox regression, the presence of maintenance steroid was associated with a lower relapse risk (Hazard ratio $=0.121, p=0.000$ ) while serum IgG4 level above twice upper limit of normal (Hazard ratio $=5.283, p=0.029$ ) and hepatobiliary involvement (Hazard ratio $=2.164, \mathrm{p}=0.095$ ) were associated with a higher relapse risk.

Conclusions: Most patients (94\%) had good response to steroid. Patients with hepatobiliary involvement and serum IgG4 level above twice upper limit of normal were at higher relapse risk and a low dose, maintenance prednisolone for longer period is recommended.

References:

[1] Kleger A, et al. IgG4-related autoimmune diseases: Polymorphous presentation complicates diagnosis and treatment. Deutsches Arzteblatt international. 2015;112(8):128-35

[2] Ghazale A, et al. Immunoglobulin G4-associated cholangitis: clinical profile and response to therapy. Gastroenterology. 2008;134(3):706-15.

[3] Kamisawa $T$, et al. Standard steroid treatment for autoimmune pancreatitis. Gut. 2009;58(11):1504-7.

Disclosure of Interest: None declared

DOI: 10.1136/annrheumdis-2017-eular.2154

\section{THU0544 THE IMPORTANCE OF R202Q POLYMORPHISM IN CLINICAL EXPRESSION OF FMF: A SINGLE CENTER CROSS-SECTIONAL STUDY}

S. Yilmaz ${ }^{1}$, E. Tekgoz ${ }^{1}$, F.I. Cinar ${ }^{2}$, M. Cinar ${ }^{1}$. ${ }^{1}$ Department of Internal Medicine, Division of Rheumatology, University of Health Sciences, Gulhane Medical Faculty; ${ }^{2}$ Gulhane School of Nursing, University of Health Sciences, Ankara, Turkey

Background: Familial Mediterranean fever (FMF) is an autosomal recessive inherited disease with recurrent fever and inflammatory episodes of serous membranes. The MEFV (Mediterranean fever) gene in the short arm of chromosome 16 is affected in the FMF. This gene encodes for a protein called 'Pyrin'. The erroneously synthesized Pyrin protein due to MEFV mutations is unable to control the post inflammatory process. Although there have been many efforts to find out genotype-phenotype association in FMF, no clear relationship has been clarified.

Objectives: In this study, the relationship between FMF clinical symptoms and MEFV gene mutations and polymorphism was investigated.

Methods: Total of 158 patients with FMF was included to the study that was conducted in a tertiary rheumatology outpatient clinic. The demographic and clinical features, as well as MEFV gene mutations were recorded in a "Patient Assessment Form". The clinical status of the disease was evaluated with FMFseverity score-2 (F-SS-2). The associations between clinical features and genetic alterations were calculated with Pearson Chi-square test.

Results: The mean age of patients was $24.3 \pm 5.1$, mean delay in diagnosis was $5.6 \pm 6.3$ years, and 155 of the patients (\%98.1) were male. The percentage of patients stating they use colchicine regularly was $136(86.1 \%)$, and mean dose was $1.4 \pm 0.3 \mathrm{mg} /$ day. The most frequent mutation was M694V $(76.6 \%)$, and $\mathrm{R} 202 \mathrm{Q}, \mathrm{M} 680 \mathrm{I}$ and $\mathrm{E} 148 \mathrm{Q}$ were found in a descending order $(60.8 \%, 19.0 \%$ and $13.9 \%$, respectively). The FMF severity score was found to be higher in patients carrying M694V mutation ( $\mathrm{p}=0.01$ ). In comparison with negative family history for FMF, M694V was more prevalent in patients with positive family history $(82.0 \%$ vs $67.2 \%, p=0.035$ ). Besides, M694V mutation found to be associated with arthritis $(p=0.045) . E 148 Q$ mutation was associated with the history of orchitis $(p=0.029)$. The most significant relation between $\mathrm{E} 148 \mathrm{Q}$ and clinical feature was family history of hemodialysis $(\mathrm{p}=0.005)$. The prevalence of non-periodic myalgia was $\% 34.2$, and this symptom was not present in patients carrying V726A mutation $(p=0.005) . M 694 V / R 202 Q$ was the most prevalent compound heterozygosis and found in 16 patients (10.1\%). This mutation (M694V/R202Q) was associated with fewer frequencies of myalgia and peritonitis, and with good response to colchicine.

Conclusions: The presence of $\mathrm{R} 202 \mathrm{Q}$ polymorphism is associated with FMF, and should be considered in the routine genetic analysis of the disease. In our patients, its co-existence with M694V seems to be associated with good response to colchicine, and to alleviate the severity of the disease expression of M694V, which is known to be associated with severe course.

\section{References:}

[1] Mor A, Shinar Y, Zaks N, Langevitz P, Chetrit A, Shtrasburg S, Rabinovitz E, Livneh A. Evaluation of disease severity in familial Mediterranean fever. Semin Arthritis Rheum. 2005 Aug;35(1):57-64 
Disclosure of Interest: None declared

DOI: 10.1136/annrheumdis-2017-eular.4294

\section{THU0545 COMPARISON OF THE CLINICAL AND LABORATORY FEATURES AT ONSET BETWEEN MULTICENTRIC CASTLEMAN'S DISEASE AND ADULT-ONSET STILL'S DISEASE}

A. González García ${ }^{1}$, W.A. Sifuentes Giraldo ${ }^{2}$, J.L. Morell Hita ${ }^{1}$, J.L. Patier de la Peña ${ }^{2} \cdot{ }^{1}$ Rheumatology; ${ }^{2}$ Internal Medicine, Ramon y Cajal University Hospital, Madrid, Spain

Background: Multicentric Castleman's disease (MCD) is a disorder characterized by polyclonal proliferation of B lymphocytes that is frequently associated with autoimmune manifestations and connective tissue diseases. MCD presents high levels of IL-6 and systemic symptoms such as fever, arthralgia, hepatosplenomegaly and serositis, so it is recommended to include MCD in the differential diagnosis of adult-onset Still's disease (AOSD). However, there are no studies comparing both groups of patients.

Objectives: To compare the clinical and laboratory features at onset between patients with MCD and AOSD seen in a Madrid tertiary care hospital.

Methods: We performed a retrospective observational study in patients with diagnosis MCD and AOSD attended our center between January 1989 and December 2015. The variables included demographics, clinical manifestations, laboratory tests and Yamaguchi's criteria.

Results: A total of 34 patients were included, 17 with MCD and 17 with AOSD. The comparison of the characteristics of both groups is presented in the table. There were no differences in age, uration of disease (MCD 158,6 days and AOSD 250,5 days, $p=0,3919$ ), diagnostic delay (MCD 18,4 and AOSD 52,2, $\mathrm{p}=0,2711$ ), arthritis, myalagias, pleuritis or macrophagic activation syndrome, but persistent fever, rash, arthralgia, pharyngitis and pericarditis were significantly more frequent in ESA, whereas male gender, human immunodeficiency virus (HIV) and/or human herpes virus 8 (HHV8) infection, hepatosplenomegaly and lymphadenopathy were significantly higher in MCD. In the laboratory test results, leukocytosis and hypertransaminasemia were significantly higher in AOSD. 52.9\% of patients with MCD met 5 or more Yamaguchi's criteria for AOSD.

\begin{tabular}{lccc}
\hline & $\begin{array}{c}\text { Multicentric Castleman's } \\
\text { disease }(\mathrm{n}=17)\end{array}$ & $\begin{array}{c}\text { Adult-onset Still's } \\
\text { disease }(\mathrm{n}=17)\end{array}$ & $\mathrm{p}$-value \\
\hline Male:female ratio & $2.4: 1$ & $0.4: 1$ & 0.0163 \\
Age at diagnosis (mean $\pm \mathrm{SD})$ & $46.4 \pm 13.1$ & $37.5 \pm 13.5$ & 0.0598 \\
HIV infection & $35.2 \%$ & $0 \%$ & 0.0154 \\
HHV8 infection & $35.2 \%$ & $0 \%$ & 0.0154 \\
Persistent fever & $47.1 \%$ & $94.1 \%$ & 0.0078 \\
Rash & $29.4 \%$ & $64.7 \%$ & 0.0392 \\
Arthralgias & $35,3 \%$ & $88.2 \%$ & 0.0014 \\
Arthritis & $0 \%$ & $17.6 \%$ & 0.0696 \\
Pharyngitis & $0 \%$ & $41.2 \%$ & 0.0029 \\
Hepatosplenomegaly & $47.1 \%$ & $5.9 \%$ & 0.0065 \\
Pericarditis & $0 \%$ & $23.5 \%$ & 0.0332 \\
Pleuritis & $17.6 \%$ & $11.7 \%$ & 0.6217 \\
Lymphadenopathy & $100 \%$ & $17.6 \%$ & 0.0001 \\
Macrophage activation syndrome & $11.7 \%$ & $17.6 \%$ & 0.6282 \\
Leucocytes $>10000 / \mathrm{mm}^{3}$ & $35.2 \%$ & $76.5 \%$ & 0.0156 \\
Increased transaminases & $0 \%$ & $58.8 \%$ & 0.0002 \\
Erythrosedimentation rate (mm/Hg) & 52 & 77.5 & 0.1104 \\
C-reactive protein (md/dl) & 75.1 & 109.5 & 0.3699 \\
Ferritin (ng/ml) & 240 & 1926 & 0.1234 \\
\hline
\end{tabular}

Conclusions: Patients with MCD may present common systemic manifestations and laboratory abnormalities at onset with AOSD and up to $50 \%$ of them may fulfill Yamaguchi's criteria for this disease, so MCD should be taken into account in its differential diagnosis.

Disclosure of Interest: None declared

DOI: 10.1136/annrheumdis-2017-eular.6228

\section{THU0546 IMPACT OF BONE LOCALISATION ON THE PROGNOSTIC OF LANGERHANS CELL HISTIOCYTOSIS: A MONOCENTRIC RETROSPECTIVE STUDY}

J.-G. Letarouilly ${ }^{1}$, N. Segaud ${ }^{1}$, B. Wallaert ${ }^{2}$, P.-Y. Hatron ${ }^{3}$, R.-M. Flipo ${ }^{1}$ ${ }^{1}$ Rhumatologie, CHRU Lille Hôpital Roger Salengro; ${ }^{2}$ Pneumologie, CHRU Lille Hôpital Calmette; ${ }^{3}$ Médecine interne, CHRU Lille Hôpital Huriez, Lille, France

Background: Langerhans cell histiocytosis $(\mathrm{LCH})$ is a rare condition, and mostly affects children. Bone is the most commonly involved organ, with bone lesions in $50 \%$ of patients. In a recent work, Arico et al. described that the probability of survival in children suffering from a multisystemic LCH with risk organ involvement was reduced if patient did not have any bony lesion [1]. There is no such a study in adult patients.

Objectives: The objective of the study was to know the bone impact on the prognosis of adult $\mathrm{LCH}$

Methods: A retrospective monocentric study was performed using data from the patients hospitalized for a LCH at Centre Hospitalier Régional Universitaire de Lille, a university hospital between 2001 and 2015. All patients with LCH and at an age of 18 years or older were included. Patients were excluded if they did not receive any osteoarticular imaging (radiography, scintigraphy, PET, MRI).

Results: Our study initially included 70 patients had LCH. After screening 54 patients met the inclusion criteria: 31 had bone localization (BLG) and 23 none (NBLG). The two groups showed differences. The lesion leading to the diagnosis was mostly osteoarticular (18 patients) in BLG and pulmonary (18 patients) in NBLG. The BLG presented more multisystemic form than the NBLG (20 vs 2, $p<0.0001)$. Both groups were equivalent for comorbidities $(p=0,206$ for cancer and $p=0,756$ for cardiovascular disease), number of smokers and age at diagnosis (36.8+/- 14.15 years vs $36.7+/-12.6$ years, $\mathrm{p}=0.96$ and 25 vs $22, \mathrm{p}=0.06$ ). Treatment was required for 14 patients in BLG and for 2 in NBLG. In BLG, 8 patients were treated medically, mostly by corticosteroid therapy and chemotherapy (7), one patient underwent radiotherapy. 6 patients were treated by surgery. In NBLG, 2 patients were treated by corticosteroid therapy. There was no surgery in NBLG (table 1). 3 patients died in BLG, 2 directly related to $L C H$ and one from postoperative infectious complications. One patient died in NBLG from hematologic pathology (chronic myelomonocytic leukemia). The time between diagnosis and death was one, six and 22 months in BLG, 36 months in NBLG. 7 patients presented relapses in BLG. 5 had been treated by medical treatment, one surgically and one had no previous treatment. The time of relapse was six, $8,12,14,16,52$ and 72 months. 4 presented new bone lesions, 1 pulmonary lesions, 1 dermatologic lesions and 1 neurologic lesion (table 1). There was no relapse in NBLG. Unfortunately, there was a lack of statistical power to conclude about the bone impact on the prognosis.

Conclusions: Patients with bone location and those with no bone location are two different phenotypes of adult LCH. Patients with bone location are more proned to have a multisystemic relapsing disease. A multicentric study with a larger number patients is needed to bring more robust data to answer about bone impact on the prognosis of adult $\mathrm{LCH}$.

\section{References:}

[1] Aricò M, Astigarraga I, Braier J, et al. Lack of bone lesions at diagnosis is associated with inferior outcome in multisystem langerhans cell histiocytosis of childhood. Br J Haematol 2015;169:241-8.

Disclosure of Interest: None declared

DOI: 10.1136/annrheumdis-2017-eular.6790

\section{THU0547 CHARACTERIZATION OF A GROUP OF 12 PATIENTS WITH MEVALONATE KINASE DEFICIENCY: SYMPTOMS AND TREATMENT WITH IL- 1 INHIBITORS}

A. Kozlova $^{1}$, V. Burlakov ${ }^{1}$, N. Kuzmenko ${ }^{1}$, S. Zimin ${ }^{2}$, T. Varlamova ${ }^{1}$, O. Barabanova ${ }^{2}$, E. Raikina ${ }^{1}$, A. Shcherbina ${ }^{1}{ }^{1}{ }^{1} /$ mmunology, Federal State Budgetary Institution "National Scientific and Practical Center of Pediatric Hematology, Oncology and Immunology named after Dmitry Rogachev" of the Ministry of Healthcare of the Russian Federation; ${ }^{2}$ Immunology, Children's Clinical Hospital N9, Moscow, Russian Federation

Background: Mevalonate kinase deficiency (MKD) is a rare autosomal recessive autoinflammatory disease caused by mutations in MVK gene. MKD patients typically have an early onset of symptoms including recurrent episodes of high fever, abdominal pain, diarrhea and vomiting, arthralgia and lymphadenopathy (AIDAI criteria for HIDS). However not all patients have typical symptoms at the time of onset. MKD treatment remains an unsolved problem, since none of the modalities previously used for MKD treatment are fully effective in the disease control.

Methods: We conducted a retrospective analysis of clinical features of twelve patients ( 6 females, 6 males) with genetically confirmed MKD. Nine patients received therapy with inhibitors of IL-1 (Anakinra and/or Canakinumab). One of the patients died from amyloidosis and macrophage activation syndrome (MAS) prior to treatment initiation, her diagnosis was verified post mortem.

Results: Ten patients had manifested within the first 6 months of life, one - at the age of 1.5 years, one - at three years of age,. During the course of the disease all patients had periodic fever and peripheral lymphadenopathy (mainly cervical group), as well as abdominal pain, nausea/vomiting. Five patients had diarrhea, sometimes with blood, one patient suffered from severe constipation. Rash was seen in eight patients, myalgia, artralgia were observed only in six. Oral ulcers were noted in seven children. Three patients had neurological involvement, one patient had it as the main symptom. One patient had periorbital edema and hyperemia during attacks, which to our knowledge, have not been reported previously in MKD. One patient developed amyloidosis and MAS before IL1 inhibitor treatment initiation, which led to her death. In patients receiving anti-IL-1 therapy AIDAl index decreased from $58.3 \pm 11,2$ before to $1,5 \pm 1,4$ after 6 month of therapy $(\mathrm{p}=0.003)$.

Conclusions: MKD symptoms can be variable and sometimes atypical, which requires physician's awareness. In our cohort of MKD patients anti IL-1 therapy was highly effective.

Disclosure of Interest: None declared

DOI: 10.1136/annrheumdis-2017-eular.2238 